N. X. Loc

Nagoya Math. J.

Vol. 54 (1974), 135-148

\title{
SUR LE PRINCIPE DU MINIMUM FIN
}

\author{
NGUYEN-XUAN-LOC
}

\section{Introduction}

Cet article fait suite à [3c]; dans [3c] étaient étudiées des généralisations éventuelles du principe de minimum fin de Fuglede (voir [2] théo. 9.1. et [3a] théo. 6.) et le cardre était celui des processus de Markov en dualité au sens de Kunita-Watanabe (voir [4b]). Le présent article a le même but, si dans [3c] on a supposé que la limite inférieure fine de la fonction finement hyperharmonique donnée doit être non négative en tout point de la fontière fine de l'ensemble de définition, on se permet dans cet article de supposer que cette lim. inf. fine pourra avoir des valeurs négatives (même égales à $-\infty$ ) sur un ensemble polaire de la fontière fine.

Les résultats principals de cet article sont la proposition 2 et les théorèmes 4 et 5 .

Nous gardons les notations et les terminologies de l'article [3c].

\section{§1. Quelques résultats complémentaires sur les balayages de potentiels}

Notons par $X=\left(\Omega, \mathscr{F}, \mathscr{F}_{t}, X_{t}, \theta_{t}, \zeta, P^{x}\right)$ un processus standard, transient sur un espace localement compact à base dénombrable (LCD) $E$.

Lemme 1. Soient $A_{1}$ et $A_{2}$ deux ensembles presque boréliens de $E$. Notons par $T_{1}$ et $T_{2}$ les temps d'entrée de $X$ dans $A_{1}$ et $A_{2}$ respectivement. Définissons la suite de temps d'arrêt $\left(\xi_{n}\right)_{n \in N}$ de la famille $\left\{\mathscr{F}_{t}\right\}_{t \geq 0}$ comme suit:

$$
\left\{\begin{aligned}
\xi_{1}=T_{1} ; \xi_{2}=T_{1}+T_{2}\left(\theta_{\xi_{1}}\right) ; \cdots \xi_{2 k}=\xi_{2 k-1}+T_{2}\left(\theta_{\xi_{2 k-1}}\right) ; \\
\xi_{2 k+1}=\xi_{2 k}+T_{1}\left(\theta_{\xi_{2 k}}\right) \\
\xi_{\infty}=\lim . \xi_{n} .
\end{aligned}\right.
$$

Alors :

a) Pour tout point $x$ de $E$ et pour tout réel $t \geqslant 0$, on $a$ :

Received January 11, 1974. 


$$
t+\xi_{\infty}\left(\theta_{t}\right) \geqslant \xi_{\infty} \quad \text { presque sûrement (p.s.) } P^{x} .
$$

b) Pour tout point $x$ irrégulier pour $A_{1}$ ou $A_{2}$ et pour toute suite $\left(t_{m}\right)_{m \geqslant 0}$ de réels décroissante vers 0 , on $a$ :

$$
\lim _{m}\left(t_{m}+\xi_{\infty}\left(\theta_{t_{m}}\right)\right)=\xi_{\infty} \quad \text { p.s. } P^{x} \text {. lorsque } m \rightarrow \infty .
$$

Démonstration. a) Voir ([3c], lemme 1).

b) Supposons que $x \in A_{1}^{\text {ir }} \cup A_{2}^{\text {ir }}$ (l'ensemble des points où soit $A_{1}$ soit $A_{2}$ est effilé), il existe alors pour le cas où $x \in A_{1}^{\text {ir }}$ un ensemble $\Omega_{0} \in \mathscr{F}_{0}$ tel que $P^{x}\left(\Omega_{0}\right)=1$ et tel que $\bigcup_{m}\left(\xi_{1}>t_{m}\right)=\Omega_{0}$ pour toute suite $\left(t_{m}\right)_{m \geqslant 0}$ de réels décroissante vers 0 .

Soient $\omega_{0} \in \Omega_{0}$ et $m_{0}$ un entier tel que $t_{m}<\xi_{1}\left(\omega_{0}\right)$ pour $m \geqslant m_{0}$, alors:

$$
t_{m}+\xi_{1}\left(\theta_{t_{m}}\left(\omega_{0}\right)\right)=\xi_{1}\left(\omega_{0}\right) \quad \text { pour } m \geqslant m_{0} \text {. }
$$

On a donc pour $m \geqslant m_{0}$ :

$$
\begin{aligned}
t_{m}+\xi_{2}\left(\theta_{t_{m}}\left(\omega_{0}\right)\right) & =t_{m}+\xi_{1}\left(\theta_{t_{m}}\left(\omega_{0}\right)\right)+T_{2}\left(\theta_{\xi_{1}}\left(\theta_{t_{m}}\left(\omega_{0}\right)\right)\right. \\
& =t_{m}+\xi_{1}\left(\theta_{t_{m}}\left(\omega_{0}\right)\right)+T_{2}\left(\theta_{t_{m}+\xi_{1}\left(\theta_{t_{m}}\right)}\left(\omega_{0}\right)\right) \\
& =\xi_{1}\left(\omega_{0}\right)+T_{2}\left(\theta_{\xi_{1}}\left(\omega_{0}\right)\right) \\
& =\xi_{2}\left(\omega_{0}\right) .
\end{aligned}
$$

Il s'ensuit par induction que $t_{m}+\xi_{k}\left(\theta_{t_{m}}\left(\omega_{0}\right)\right)=\xi_{k}\left(\omega_{0}\right)$ pour tout entier $k$ et pour tout $m \geqslant m_{0}$. D'autre part on a d'après a):

$$
\begin{aligned}
\xi_{\infty}\left(\omega_{0}\right) & =\lim _{k} \xi_{k}\left(\omega_{0}\right)=\lim _{k}\left(t_{m}+\xi_{k}\left(\theta_{t_{m}}\left(\omega_{0}\right)\right) \quad m \geqslant m_{0}\right. \\
& =t_{m}+\xi_{\infty}\left(\theta_{t_{m}}\left(\omega_{0}\right)\right) \\
& \geqslant \xi_{\infty}\left(\omega_{0}\right) .
\end{aligned}
$$

Il reste à montrer (2) pour tout point $x$ de l'ensemble $A_{2}^{\text {ir }} \backslash A_{1}^{\text {ir. }}$. Si $x$ est régulier pour $A_{1}$ il existe d'abord un ensemble $\Omega_{0} \in \mathscr{F}_{0}$ tel que $P^{x}\left(\Omega_{0}\right)=1$ et tel que:

$$
\lim _{m}\left(t_{m}+\xi_{1}\left(\theta_{t_{m}}\left(\omega_{0}\right)\right)=\xi_{1}\left(\omega_{0}\right)=0 \quad \text { pour tout } \omega_{0} \in \Omega_{0} .\right.
$$

Puisque $x$ est aussi irrégulier pour $A_{2}$ on a p.s. $P^{x}$ sur $\Omega_{0}$ :

$$
t+T_{2}\left(\theta_{t}\left(\omega_{0}\right)\right)=T_{2}\left(\omega_{0}\right) \quad \text { pour tout } 0 \leqslant t<t_{m_{0}}
$$

où $m_{0}$ est un entier dépendant de $\omega_{0}$.

Il suffit donc de choisir un entier $m_{1}$ tel que $t_{m}+\xi_{1}\left(\theta_{t_{m}}\left(\omega_{0}\right)\right)<t_{m_{0}}$ pour tout $m \geqslant m_{1}$ pour voir que: 


$$
\begin{aligned}
& t_{m}+\xi_{2}\left(\theta_{t_{m}}\left(\omega_{0}\right)\right)=t_{m}+\xi_{1}\left(\theta_{t_{m}}\left(\omega_{0}\right)\right)+T_{2}\left(\theta_{t_{m+\hat{\xi}_{1}\left(\theta_{t_{m}}\right)}}\left(\omega_{0}\right)\right) \quad m \geqslant m_{1} \\
& =T_{2}\left(\omega_{0}\right) \\
& =\xi_{1}\left(\omega_{0}\right)+T_{2}\left(\theta_{\hat{\xi}_{1}}\left(\omega_{0}\right)\right)=\xi_{2}\left(\omega_{0}\right) \text {. }
\end{aligned}
$$

La suite de la démonstration se fait exactement comme celle du cas précédent.

Proposition 2. Soit $\hat{X}=\left(\hat{\Omega}, \hat{\mathscr{F}}, \hat{\mathscr{F}}_{t}, \hat{X}_{t}, \hat{\theta}_{t}, \hat{\zeta}, \hat{P}^{x}\right)$ un second processus standard et transient sur $E$ et soient $A_{1}$ et $A_{2}$ deux ensembles presque boréliens de $E$. Supposons que $X$ et $\hat{X}$ sont en dualité dans le sens de Kunita-Watanabe et notons par $u(x, y)$ une fonction de Green de $X$. Définissons la suite de temps d'arrêt $\left(\hat{\xi}_{n}\right)_{n \in N}$ comme suit:

$$
\left\{\begin{aligned}
\hat{\xi}_{1}=\hat{T}_{2} ; \hat{\xi}_{2}=\hat{\xi}_{1}+\hat{T}_{1}\left(\hat{\theta}_{\hat{\xi}_{1}}\right) ; \cdots \hat{\xi}_{2 k}=\hat{\xi}_{2 k-1}+\hat{T}_{1}\left(\hat{\theta}_{\hat{\xi}_{2 k-1}}\right) ; \\
\hat{\xi}_{2 k+1}=\hat{\xi}_{2 k}+\hat{T}_{2}\left(\hat{\theta}_{\hat{\xi}_{2 k}}\right) \\
\hat{\xi}_{\infty}=\lim _{k} \hat{\xi}_{k}
\end{aligned}\right.
$$

où $\hat{T}_{1}$ et $\hat{T}_{2}$ dénotent respectivement les temps d'entrée de $\hat{X}$ dans $A_{1}$ et $A_{2}$. Alors:

a) On a pour tout point $x$ de $A_{1}^{\mathrm{ir}} \cup A_{2}^{\mathrm{ir}} \cup\left(A_{1} \cap A_{2}\right)^{\mathrm{r}}$ :

(4) $u \hat{P}_{\hat{\xi}_{\infty}}(x, y)=P_{\xi_{\infty}} u(x, y) \quad$ pour tout $y \in A_{1}^{\text {coir }} \cup A_{2}^{\text {coir }} \cup\left(A_{1} \cap A_{2}\right)^{\text {cor }}$ et vice-versa on a pour tout $y$ de $A_{1}^{\text {coir }} \cup A_{2}^{\text {coir }} \cup\left(A_{1} \cap A_{2}\right)^{\text {cor }}$ :

$$
u \hat{P}_{\hat{\xi}_{\infty}}(x, y)=P_{\xi_{\infty}} u(x, y) \quad \text { pour tout } x \text { de } A_{1}^{\mathrm{ir}} \cup A_{2}^{\mathrm{ir}} \cup\left(A_{1} \cap A_{2}\right)^{\mathrm{r}} .
$$

b) Pour tout potentiel de Green $p(x)=\int u(x, y) \cdot r(d y)$ dont la mesure associée $r(d y)$ ne charge pas les sous-ensembles semi-polaires de $A_{1}^{\text {cor }} \cap$ $A_{2}^{\text {cor }} \backslash\left(A_{1} \cap A_{2}\right)^{\text {cor }}$

$$
\lim _{n} E_{x}\left(p\left(X_{\xi_{n}}\right) ; \xi_{n}<\zeta\right)=E_{x}\left(p\left(X_{\xi_{\infty}}\right) ; \xi_{\infty}<\zeta\right)
$$

pour tout $x$ en dehors un ensemble semi-polaire (q.p.) de $\left(A_{1} \cap A_{2}\right)^{\mathrm{r}} \cup$ $A_{1}^{\text {ir }} \cup A_{2}^{\text {ir }}$.

De plus si $A_{1}$ et $A_{2}$ sont finement fermés et si $p$ est fini dans $\left(E \backslash A_{1} \cap A_{2}\right.$ ) alors on a l'égalité (5) pour tout $x$ de ce dernier ensemble.

Démonstration. Rappelons que pour tout temps d'arrêt $T$ (resp. $\hat{T}$ ) de la famille $\left\{\mathscr{F}_{t}\right\}_{t \geqslant 0}$ (resp. $\left\{\hat{\mathscr{F}}_{t}\right\}_{t \geqslant 0}$ ) et pour toute fonction presque borélienne non négative $f, P_{T} f$ (resp. $\hat{P}_{\hat{T}} f$ ) dénote le balayé (resp. cobalayé) de $f$ pour $X$ (resp. pour $\hat{X}$ ). Ainsi : 


$$
\begin{array}{ll}
P_{\xi_{n}} u(x, y):=E_{x}\left(u\left(X_{\xi_{n}}, y\right) ; \xi_{n}<\zeta\right) & (n=1,2,3, \cdots) \\
u \hat{P}_{\xi_{n}}(x, y):=\hat{E}_{y}\left(u\left(x, \hat{X}_{\hat{\xi}_{n}}\right) ; \hat{\xi}_{n}<\hat{\zeta}\right) & (n=1,2,3, \cdots)
\end{array}
$$

a) D'après ([1], (V.1.16)) :

$$
\begin{array}{ll}
P_{T_{1}} u(x, y)=u \hat{P}_{\hat{T}_{1}}(x, y) & \text { pour tout }(x, y) \in E \times E, \\
P_{T_{2}} u(x, y)=u \hat{P}_{\hat{T}_{2}}(x, y) & \text { pour tout }(x, y) \in E \times E .
\end{array}
$$

Donc:

$$
\begin{aligned}
P_{T_{2}}\left(P_{T_{1}} u\right)(x, y) & =\int P_{T_{2}}(x, d z) \int u(z, r) \cdot \hat{P}_{\hat{T}_{1}}(d r, y) \\
& =\int u \hat{P}_{\hat{T}_{2}}(x, r) \cdot \hat{P}_{\hat{T}_{1}}(d r, y) \\
& =\left(u \hat{P}_{\hat{T}_{2}}\right) \hat{P}_{\hat{T}_{1}}(x, y) .
\end{aligned}
$$

On a par induction sur $k$ :

$$
P_{\xi_{2 k}} u(x, y)=u \hat{P}_{\hat{\xi}_{2 k}}(x, y) \quad \text { pour tout }(x, y) \in E \times E .
$$

Soient $f$ et $g$ deux fonctions boréliennes, non négatives, bornées quelconques dans $E$, posons $\nu(d x):=f(x) m(d x)(m(d x)$ dénote la mesure de référence de l'hypothèse de Kunita-Watanabe) et $\mu(d y):=g(y) m(d y)$, puisque

$$
f \hat{U}(\cdot)=\int u(x, \cdot) f(x) m(d x) \quad \text { et } \quad U g(\cdot)=\int u(\cdot, y) g(y) m(d y)
$$

sont des potentiel (co-)réguliers sur $E$, on a donc:

$$
\begin{aligned}
\iint u \hat{P}_{\hat{\xi}_{\infty}}(x, y) f(x) g(y) m(d x) m(d y) & =\hat{E}_{\mu}\left(f \hat{U}\left(\hat{X}_{\hat{\xi}_{\infty}}\right) ; \hat{\xi}_{\infty}<\hat{\zeta}\right) \\
& =\lim _{k} \hat{E}_{\mu}\left(f \hat{U}\left(\hat{X}_{\hat{\xi}_{2 k}}\right) ; \hat{\xi}_{2 k}<\hat{\zeta}\right)
\end{aligned}
$$

et

$$
\begin{aligned}
\iint P_{\xi_{\infty}} u(x, y) f(x) g(y) m(d x) m(d y) & =E_{\nu}\left(U g\left(X_{\xi_{\infty}}\right) ; \xi_{\infty}<\zeta\right) \\
& =\lim _{k} E_{\nu}\left(U g\left(X_{\xi_{2 k}}\right) ; \xi_{2 k}<\zeta\right)
\end{aligned}
$$

D'autre part d'après (6):

$$
E_{\nu}\left(U g\left(X_{\xi_{2 k}}\right) ; \xi_{2 k}<\zeta\right)=\hat{E}_{\mu}\left(f \hat{U}\left(\hat{X}_{\hat{\xi}_{2 k}}\right) ; \hat{\xi}_{2 k}<\hat{\zeta}\right) \quad(k=1,2,3 \cdots)
$$

Ce qui implique:

$$
u \hat{P}_{\hat{\xi}_{\infty}}(x, y)=P_{\xi_{\infty}} u(x, y) \quad \text { p.s. } m(d x) \times m(d y) \text { sur } E \times E .
$$


Ainsi nous avons pour tout $x$ en dehors un certain ensemble de mesure $m(d x)$ nulle:

$$
u \hat{P}_{\hat{\xi}_{\infty}}(x, y)=P_{\xi_{\infty}} u(x, y) \quad \text { p.s. } m(d y) \text { sur } E .
$$

D'après le lemme 1 le temps d'arrêt $\hat{\xi}_{\infty}$ de la famille $\left(\hat{\mathscr{F}}_{t}\right)_{t \geqslant 0}$ satisfait à la propriété $t+\hat{\xi}_{\infty}\left(\hat{\theta}_{t}\right) \geqslant \hat{\xi}_{\infty}$ p.s. $P^{y}(y \in E, t \geqslant 0)$, la fonction

$$
y \rightarrow u \hat{P}_{\hat{\xi}_{\infty}}(x, y)
$$

est donc cosurmédiane, càd, surmédiane pour le semi-groupe associé à $\hat{X}$. D'autre part la fonction $y \rightarrow P_{\xi \infty} u(x, y)$ est coexcessive, d'après (7) elle est donc la régularisation excessive de $y \rightarrow u \hat{P}_{\hat{\xi}_{\infty}}(x, y)$, ce qui implique ([4a], IX.T66) :

(8) $\lim _{t}\left(u \hat{P}_{\hat{\xi}_{\infty}}(x, \cdot)\right) \cdot \hat{P}_{t}(y)=P_{\hat{\xi}_{\infty}} u(x, y) \quad$ pour tout $y \in E$ lorsque $t \downarrow 0$.

D'une part on a d'après le lemme 1 pour tout point $y$ de $A_{1}^{\text {coir }} \cup A_{2}^{\text {coir }}$ et pour toute suite de réels $\left(t_{m}\right)$ décroissante vers 0 :

$$
\begin{aligned}
\lim _{m}\left(u \hat{P}_{\hat{\xi}_{\infty}}(x, \cdot)\right) \cdot \hat{P}_{t_{m}}(y) & =\lim _{m} \hat{P}_{t_{m}+\hat{\xi}_{\infty}\left(\hat{\theta}_{t_{m}}\right)}(x, y) \\
& =u \hat{P}_{\hat{\xi}_{\infty}}(x, y) .
\end{aligned}
$$

D'autre part si $y$ appartient à $\left(A_{1} \cap A_{2}\right)^{\text {cor }}$, alors $0 \leqslant \hat{\xi}_{\infty} \leqslant \hat{T}_{A_{1} \cap A_{2}}=0$ p.s. $P^{y}$ et $u \hat{P}_{\hat{\xi}_{\infty}}(x, y)=u(x, y)$. Remarquons d'abord que l'intersection de tout ensemble cofinement dense dans $E$ avec l'ensemble $\left(A_{1} \cap A_{2}\right)^{\text {cor }}$ est aussi cofinement dense dans ce dernier ensemble puisque l'ensemble $\left(A_{1} \cap A_{2}\right)^{\text {cor }} \backslash$ intérieur-cofine $\left(A_{1} \cap A_{2}\right)$ est contenu dans la frontière cofine de $A_{1} \cap A_{2}$. Ainsi les deux fonctions $y \rightarrow u \hat{P}_{\hat{\xi}_{\infty}}(x, y)$ et $y \rightarrow P_{\xi_{\infty}} u(x, y)$ sont cofinement continues dans $\left(A_{1} \cap A_{2}\right)^{\text {cor }}$ et $y$ sont égales sur un sous ensemble dense pour la topologie cofine (le complémentaire d'un ensemble de mesure $m(d y)$ nulle), donc elles sont partout égales sur $\left(A_{1} \cap A_{2}\right)^{\text {cor. }}$. On a ainsi montré que pour p.s. $m(d x)$ dans $E$ :

$$
\left\{y \mid P_{\hat{\xi}_{\infty}} u(x, y)=u \hat{P}_{\hat{\xi}_{\infty}}(x, y)\right\} \supset\left(A_{1} \cap A_{2}\right)^{\text {cor }} \cup\left(E \backslash A_{1}^{\text {cor }}\right) \cup\left(E \backslash A_{2}^{\text {cor }}\right)
$$

càd (4). Par dualité on a aussi pour $y$ en dehors d'un ensemble de mesure $m(d y)$ nulle dans $E$ :

$$
P_{\xi_{\infty}} u(x, y)=u \hat{P}_{\hat{\xi}_{\infty}}(x, y) \quad \text { pour tout } x \in\left(A_{1} \cap A_{2}\right)^{\mathrm{r}} \cup\left(E \backslash A_{1}^{\mathrm{r}}\right) \cup\left(E \backslash A_{2}^{\mathrm{r}}\right) .
$$

Pour un tel $x$ on utilise de nouveau l'argument indiqué en haut pour montrer (4) et on achève la démonstration de cette partie de la proposition par un argument de dualité. 
b) Il suffit de montrer (5) pour le cas où $r(d y)$ est une mesure de Radon non-négative et bornée de $E$. Nous esquissons un argument déjà utilisé dans la démonstration de ([3c], lemme 3).

La suite de fonctions $X$-excessives:

$$
u_{n}(x)=E_{x}\left(p\left(X_{\xi_{n}}\right) ; \xi_{n}<\zeta\right) \quad(n=1,2,3 \cdots)
$$

décroit p.s. $m(d x)$ vers la régularisation excessive $\hat{u}(x)$ de $\inf _{n} u_{n}(x):=$ $u(x)$. Posons $r_{n}(d y):=\hat{P}_{\hat{\xi}_{n}} r(d y)$, alors

$$
u_{n}(x)=\int u(x, y) \cdot r_{n}(d y)
$$

D'autre part puisque la suite de mesures $\left(r_{n}(d y)\right)_{n \in N}$ converge vaguement vers la mesure $\hat{P}_{\hat{\xi}_{\infty}} r(d y)$ on a d'après ([4b], III.T.8):

$$
\begin{aligned}
\hat{u}(x) & =\int u(x, y) \hat{P}_{\hat{\xi}_{\infty}} r(d y) \\
& =\int r(d y) u \hat{P}_{\hat{\xi}_{\infty}}(x, y) .
\end{aligned}
$$

L'ensemble $\left\{y \mid u \hat{P}_{\hat{\xi}_{\infty}}(x, y)>P_{\xi_{\infty}} u(x, y)\right\}$ est semi-polaire donc si $r(d y)$ ne charge pas les sous-ensembles semi-polaires de $A_{1}^{\text {cor }} \cap A_{2}^{\text {cor }} \backslash\left(A_{1} \cap A_{2}\right)^{\text {cor }}$ on a d'après (4): Pour tout $x$ de $\left(A_{1} \cap A_{2}\right)^{\mathrm{r}} \cup\left(E \backslash A_{1}^{\mathrm{r}}\right) \cup\left(E \backslash A_{2}^{\mathrm{r}}\right)$

$$
\hat{u}(x)=\int r(d y) P_{\xi_{\infty}} u(x, y)=P_{\xi_{\infty}} p(x) .
$$

L'égalité (5) est donc vrai pour tout $x$ de $\{\hat{u}=u\} \cap\left\{\left(A_{1} \cap A_{2}\right)^{\mathrm{r}} \cup\left(E \backslash A_{1}^{\mathrm{r}}\right)\right.$ $\left.\cup\left(E \backslash A_{2}^{\mathrm{r}}\right)\right\}$. Si de plus $A_{1}$ et $A_{2}$ sont finement fermés on a au moins l'égalité (5) pour tout $x$ de $\{\hat{u}=u\} \cap\left(E \backslash A_{1} \cap A_{2}\right)$. D'autre part pour tout $x$ de $\left(E \backslash A_{1} \cap A_{2}\right)$ on a $\hat{u}(x)=u(x)$ puisque $p(x)$ est fini et puisque $\xi_{n}>0$ p.s. $P^{x}$ pour $n \geqslant 2$.

Remarques. 1) Pour tout $x$ de $E$ tel que $p(x)<+\infty$ la suite de variables aléatoires $\left\{p\left(X_{\xi_{n}}\right) \cdot X_{\left\{\xi_{n}<\xi\right\}}\right\}_{n \in N}$ est un supermartingale non-négative de la famille $\left\{\mathscr{F}_{\xi_{n}}\right\}_{n \in N}$, on a donc:

$$
\lim _{n} p\left(X_{\xi_{n}}\right) \cdot X_{\left[\xi_{n}<\xi\right]}=Y(\cdot) \quad \text { p.s. } P^{x} \text {. }
$$

où $Y(\cdot)$ est une fonction $P^{x}$-intégrable. Donc pour tout point $x$ de $\{p<+\infty\} \cap\{\hat{u}=u\} \cap\left\{\left(A_{1} \cap A_{2}\right)^{\mathrm{r}} \cup\left(E \backslash A_{1}^{\mathrm{r}}\right) \cup\left(E \backslash A_{2}^{\mathrm{r}}\right)\right\}$ on a d'après (5) : 


$$
\begin{aligned}
E_{x}\left(p\left(X_{\xi_{\infty}}\right) ; \xi_{\infty}<\zeta\right) & =\lim _{n} E_{x}\left(p\left(X_{\xi_{n}}\right) ; \xi_{n}<\zeta\right) \\
& \geqq E_{x}(Y(\cdot)) \\
& \geqq E_{x}\left(p\left(X_{\xi_{\infty}}\right) ; \xi_{\infty}<\zeta\right),
\end{aligned}
$$

càd $\left\{p\left(X_{\xi_{n}}\right) \cdot X_{\left\{\hat{\xi}_{n}<\xi\right\}}\right\}_{n \in N}$ est un surmartingale uniformément intégrable.

2) Si $r(d y)$ ne charge pas les ensembles semi-polaires de $E$ (ce qui est équivalent dans le cas classique à dire que $p$ est semi-borné) la démonstration de (5) se simplifie beaucoup et on peut même montrer que le surmatingale $\left\{\left(p\left(X_{t}\right)\right)_{t \geqslant 0}, P^{x}\right\}$ de la famille $\left\{\mathscr{F}_{t}\right\}_{t \geqslant 0}$ est de classe (D) pour quasi-partout $x$ dans $E$. Nous avons supposé dans le lemme 3 de [3c] que $p$ est fini sur la fermeture cofine de $\left(E \backslash A_{1} \cap A_{2}\right)$ et cette condition est plus forte que celle de la proposition 2 car elle entraine que $r(d y)$ ne charge pas des ensembles polaires de la fermeture cofine de $\left(E \backslash A_{1} \cap\right.$ $A_{2}$ ) (voir [3b]). Le contre-exemple suivant montre que notre condition sur la mesure $r(d y)$ dans la proposition 2 est la meilleure que nous puissent obtenir: Soit $X$ le mouvement brownien dans $R^{3}$. Soient $B$ la boule unité ouverte et $B_{1}, B_{2}$ deux boules fermées tangent à l'origine. Posons $A_{1}:=B_{1} \cup \complement B, A_{2}:=B_{2} \cup \complement B$ et $p(x)=|x|^{-1}$. Alors $A_{1}^{\text {cor }} \cap A_{2}^{\text {cor }} \backslash$ $\left(A_{1} \cap A_{2}\right)^{\text {cor }}=\{0\}$ et la mesure $r(d y)$ est la masse unitée à l'origine. Il est facile de voir que

$$
E_{x}\left(p\left(X_{\xi_{n}}\right) ; \xi_{n}<\zeta\right)=p(x) \quad \text { pour tout } x \text { de } E . \quad(n=1,2,3 \cdots)
$$

et

$$
\begin{aligned}
E_{x}\left(p\left(X_{\xi_{\infty}}\right) ; \xi_{\infty}<\zeta\right)= & P_{T C_{B}}(x) \quad \text { pour tout } x \text { de } E, \\
& <p(x) \quad \text { pour tout } x \text { de } B .
\end{aligned}
$$

\section{§ 2. Le principe du minimum fin}

Nous appelons comme dans [3c] le cas $\left(B_{2}\right)$ l'ensemble des hypothèses suivantes:

(-Dualité au sens de Kunita-Watanabe de deux processus standards, transients $X$ et $\hat{X}$.

-Les trajectoires de $X$ sont continues.

_Les ensembles semi-polaires sont polaires.

Rappelons aussi que pour une fonction numérique. u.m. $f$ sur $E$ et pour un temps d'arrêt $T$ de la famille $\left\{\mathscr{F}_{t}\right\}_{t \geqslant 0}$ tel que $P_{T} f^{-}(x)<+\infty$, nous définissons $E_{x}\left(f\left(X_{T}\right) ; T<\zeta\right)$ comme l'intégrale supérieure de la fonction $f\left(X_{T}\right)$ par rapport à la mesure $P^{x}$. 
LEMme 3. Etant donné le cas $\left(B_{2}\right)$. Soit $f: E \rightarrow[-\infty,+\infty]$ une fonction finement semi-continue inférieurement (s.c.i.) et à valeurs q.p. plus grandes que $-\infty$ dans $E$. Soient $\left(A_{i}\right)_{i \in N}$ une suite d'ensembles finement fermés et notons par $A$ l'ensemble $\bigcap_{i=1}^{\infty} A_{i}$.

Supposons que:

1) $\quad f(x) \geqslant E_{x}\left(f\left(X_{T_{A_{i}}}\right) ; T_{A_{i}}<\zeta\right)$ pour q.p. $x$ dans $E \backslash A_{i}(i=1,2 \ldots)$

2) $f \geqslant-p$ dans $U:=E \backslash A$ où $p(x)=\int u(x, y) r(d y)$ est un potentiel de Green fini dans $U$.

3) $\quad r(d y)$ ne charge pas les sous-ensembles polaires de $\partial_{\text {cof }} A \backslash A^{\text {cor }}$, où $\partial_{\text {cof }} A$ dénote la fontière cofine de $A$.

Alors:

$$
f(x) \geqslant E_{x}\left(f\left(X_{T_{A}}\right) ; T_{A}<\zeta\right) \quad \text { q.p. dans } U \text {. }
$$

Démonstration. Considérons d'abord les ensembles $A_{1}$ et $A_{2}$ et la suite $\left(\xi_{n}\right)_{n \in N}$ de temps d'arrêt définie par (1), alors $r(d y)$ ne charge pas les sous-ensembles polaires de $A_{1}^{\text {cor }} \cap A_{2}^{\text {cor }} \backslash\left(A_{1} \cap A_{2}\right)^{\text {cor. }}$. En effet ce dernier ensemble n'est autre que $\partial_{\text {cof }}\left(A_{1} \cap A_{2}\right) \backslash\left(A_{1} \cap A_{2}\right)^{\text {cor }} ; p$ est fini dans $U$ donc $r(d y)$ ne charge pas les sous-ensembles polaires de $U$ ([3]), à fortiori, ceux de $\left\{\partial_{\text {cof }}\left(A_{1} \cap A_{2}\right) \backslash\left(A_{1} \cap A_{2}\right)^{\text {cor }}\right\} \cap$. D'autre part d'après (3) $r(d y)$ ne charge pas aussi les sous-ensembles polaires de $\left\{\partial_{\text {cof }}\left(A_{1} \cap A_{2}\right) \backslash\left(A_{1} \cap\right.\right.$ $\left.A_{2}\right)^{\text {cor }} \cap \cap\left(\partial_{\text {cor }} A \backslash A^{\text {cor }}\right)$, il nous reste à montrer que $\left\{\partial_{\text {cof }}\left(A_{1} \cap A_{2}\right) \backslash\left(A_{1} \cap A_{2}\right)^{\text {corr }}\right\}$ $\cap\left(\partial_{\text {cof }} A \cap A^{\text {cor }}\right)$ est vide et ce fait est évident car si $y$ est un point de ce dernier ensemble il est donc corégulier pour $A$, à fortiori, pour $A_{1} \cap$ $A_{2}$, ce qui est impossible. Soit $x$ un point de $\left(E \backslash A_{1} \cap A_{2}\right) \subset U, p(x)$ est donc fini, alors d'après la proposition $2\left\{p\left(X_{\xi_{n}}\right) \cdot X_{\left\{\xi_{n}<\xi\right\}}, P^{x}\right\}$ est un surmartingale uniforme avec limite $p\left(X_{\xi_{\infty}}\right)$. D'après (2), le lemme de Fatou et ([3c], 1.e.), nous avons:

$$
\begin{aligned}
\liminf _{n} E_{x}\left(f\left(X_{\xi_{n}}\right) ; \xi_{n}<\zeta\right) & \geqslant E_{x}\left(\liminf _{n} f\left(X_{\xi_{n}}\right) \xi_{n}<\zeta\right) \\
& =E_{x}\left(f\left(X_{\xi_{\infty}}\right) ; \xi_{\infty}<\zeta\right)
\end{aligned}
$$

D'autre part d'après la propriété de Markov forte de $X$ :

$$
f(x) \geqslant E_{x}\left(f\left(X_{\xi_{n}}\right) ; \xi_{n}<\zeta\right) \quad(n=1,2,3 \cdots)
$$

ce qui implique:

$$
f(x) \geqslant E_{x}\left(f\left(X_{\xi_{\infty}}\right) ; \xi_{\infty}<\zeta\right) \quad \text { pour tout } x \text { dans } U .
$$


Un argument analogue à celui de la démonstration de ([3c], lemme 4) montre que:

$$
f(x) \geqslant E_{x}\left(f\left(X_{T_{A_{1} \cap A_{2}}}\right) ; T_{A_{1} \cap A_{2}}<\zeta\right) \quad \text { q.p. } x \text { dans } U .
$$

En remarquant que $r(d y)$ ne charge pas $\left\{\partial_{\text {cof }}\left(\cap_{i=1}^{n} A_{i}\right) \backslash\left(\cap_{i=1}^{n} A_{i}\right)^{\text {cor }}\right\}(n \in N$ $\cup\{\infty\})$ on peut utiliser les arguments de la démonstration de ([3c], théo. 6) pour montrer (9).

THÉORÈME 4. Supposons $\left(B_{2}\right)$. Soit $f: U \rightarrow[-\infty,+\infty]$ une fonction définie sur un ensemble finement ouvert $U$ de $E$, telle que:

1) $f$ est finement s.c.i. et $>-\infty$.

2) $f$ peut être prolongée sur $E$ en une fonction $\vec{f}, q . p .>-\infty$ et $q . p$. finement s.c.i.

3) il existe une base $\left(V_{\alpha}\right)_{\alpha \in I}$ de la topologie fine de $U$ consistant des ensembles finement ouverts telle que; Pour tout $\alpha \in I$ :

$$
f(x) \geqslant E_{x}\left(\bar{f}\left(X_{\tau_{\alpha}}\right) ; \tau_{\alpha}<\zeta\right) \quad \text { pour tout } x \text { de } V_{\alpha}
$$

où $\tau_{\alpha}:=T_{E \backslash \bigvee \alpha}(\alpha \in I)$.

4) $f \geqslant-p$ dans $U$, ò̀ $p(x):=\int u(x, y) r(d y)$ est un potentiel de Green fini dans $U$.

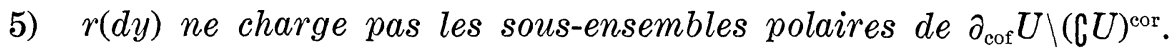

Alors:

$$
f(x) \geqslant E_{x}\left(\bar{f}\left(X_{\tau_{U}}\right) ; \tau_{U}<\zeta\right) \quad \text { pour q.p. } x \text { dans } U .
$$

où $\tau_{U}:=T_{E \backslash U}$.

En particulier si la condition 2) est remplacée par:

$$
\liminf _{x \in U, x \rightarrow y} f(x) \geqslant 0 \quad \text { pour q.p. } y \text { de } \partial_{f} U \text {. }
$$

Alors $f$ est non-négative dans $U$.

Démonstration. $\bar{f}$ est q.p. finement s.c.i. dans $E$ et les trajectoires de $X$ sont continues, d'après ([3c], 1.e.): Pour toute loi de probabilité $\mu$ sur $E$, la fonction $t \rightarrow \vec{f}\left(X_{t}(\omega)\right)$ est continue pour la topologie gauche sur ]0, $\zeta(\omega)\left[\right.$ p.s. $P^{\mu}$.

D'autre part puisque la topologie fine est quasi-lindelöf il existe une suite $\left(V_{i}\right)_{i \in N}$ d'éléments de $\left(V_{\alpha}\right)_{\alpha \in I}$ telle que:

$$
\bigcup_{i=1}^{\infty} V_{i}=U \backslash e_{0}:=U^{\prime} \quad \text { où } e_{0} \text { est un sous-ensemble polaire de } U \text {. }
$$


$\partial_{\text {cof }} U^{\prime}$ est contenue dans $\partial_{\text {cof }} U \cup e_{0}$; en effet soit $y$ un point de $\partial_{\text {cor }} U^{\prime} \backslash e_{0}$ et soit $W$ un voisinage cofine quelconque de $y$, alors d'une part $W \cap U$ $\supset W \cap U^{\prime} \neq \emptyset$ et d'autre part $W \cap(E \backslash U)=\left(W \backslash e_{0}\right) \cap\left(E \backslash U^{\prime}\right)$ est aussi non-vide puisque $\left(W \backslash e_{0}\right)$ est un voisinage cofine de $y$. Montrons en suite que $r(d y)$ ne charge pas les sous-ensembles polaires de $\partial_{\text {cof }} U^{\prime} \backslash\left(\complement U^{\prime}\right)^{\text {cor }}$. On a:

$$
\begin{aligned}
& \partial_{\text {cof }} U^{\prime} \backslash\left(\mathcal{C} U^{\prime}\right)^{\text {cor }} \subseteq\left\{\partial_{\text {cof }} U \cup e_{0}\right\} \backslash\left(\mathcal{C} U^{\prime}\right)^{\mathrm{cor}} \\
& \subseteq\left\{\partial_{\text {cof }} U \backslash\left(\complement U^{\prime}\right)^{\mathrm{cor}}\right\} \cup\left\{e_{0} \backslash\left(\complement U^{\prime}\right)^{\mathrm{cor}}\right\}
\end{aligned}
$$

$p$ est fini sur $U$, donc $r(d y)$ ne charge pas les sous-ensembles polaires de $U$, à fortiori, $r(d y)$ ne charge pas l'ensemble polaire $e_{0} \backslash\left(E \backslash U^{\prime}\right)^{\mathrm{cor}}$; d'autre part puisque $\left(E \backslash U^{\prime}\right)^{\text {cor }} \equiv(E \backslash U)^{\text {cor }}$, d'après (5) $r(d y)$ ne charge pas aussi les sous-ensembles polaires de $\partial_{\text {cof }} U \backslash\left(\mathcal{C} U^{\prime}\right)^{\text {cor }}$. Posons maintenant $A_{i}:=E \backslash V_{i}(i=1,2,3 \cdots)$, alors:

$$
A_{i}:=\bigcap_{i=1}^{\infty} A_{i}=U^{\prime} \quad \text { et } \quad \partial_{\mathrm{cof}} A \backslash A^{\mathrm{cor}} \equiv \partial_{\mathrm{cof}} U^{\prime} \backslash\left(\mathcal{C} U^{\prime}\right)^{\mathrm{cor}} .
$$

Le lemme 3, appliqué à $\bar{f}$ et $\left(A_{i}\right)_{i \in N}$, donne donc:

$$
\left\{\begin{aligned}
f(x)=\bar{f}(x) \geqslant E_{x}\left(\bar{f}\left(X_{T_{A}}\right) ; T_{A}<\zeta\right) & \text { pour q.p. } x \text { dans } E \backslash A \\
:=E_{x}\left(\bar{f}\left(X_{\tau_{U}}\right) ; \tau_{U}<\zeta\right) & \text { pour q.p. } x \text { dans } U^{\prime}
\end{aligned}\right.
$$

Supposons maintenant que:

$$
\liminf _{x \in U, x \rightarrow y} f(x) \geqslant 0 \quad \text { pour q.p. } y \text { de } \partial_{f} U \text {. }
$$

Alors $\bar{f}$ peut être choisir comme la fonction égale à $f$ dans $U$ et égale à 0 dans $E \backslash U$. Pour tout $x \in U, X_{\tau_{U}}(\omega)$ appartient p.s. $P^{x}$ à $\partial_{f} U$, (11) donne:

$$
\left\{\begin{array}{lll}
f(x) \geqslant E_{x}\left(\bar{f}\left(X_{\tau_{U}}\right) ; \tau_{U}<\zeta\right) & \text { q.p. } x \in U, \\
& =0 & \text { q.p. } x \in U .
\end{array}\right.
$$

Soit $x_{0}$ un point de $U$ tel que (12) n'est pas vérifiée, prenons un $V_{\alpha_{0}}$ de $\left(V_{\alpha}\right)_{\alpha \in I}$ tel que $x_{0} \in V_{\alpha_{0}}$ alors :

$$
\begin{aligned}
f\left(x_{0}\right) & \geqslant E_{x_{0}}\left(\bar{f}\left(X_{\tau_{\nabla_{\alpha_{0}}}}\right) ; \tau_{V_{\alpha_{0}}}<\zeta\right) \\
& =0 .
\end{aligned}
$$

Dans la suite nous considérons toujours des espaces harmoniques $(\mathscr{H}, E)$ du type de Bauer tels que: 
-Il existe un potentiel strictement positif dans $E$.

$-E$ est un (LCD) et la fonction constante 1 est hyperharmonique.

-Une fonction de Green pour $(\mathscr{H}, E)$ existe.

-Les ensembles semi-polaires sont polaires.

Sous ces conditions on peut montrer qu'il existe un couple de processus de Hunt $(X, \hat{X})$ en dualité dans le sens de Kunita-Watanabe tel que $X$ est une diffusion associée à ( $\mathscr{H}, E$ ) (voir [3c], 4.c); nous avons donc le $\operatorname{cas}\left(B_{2}\right)$.

DÉFINITION ([2]). Soit $U$ un ouvert fin de $E$. Une fonction numérique $f$ définie sur $U$ est appelée finement hyperharmonique dans $U$ si:

(i) $f$ est $>-\infty$, finement s.c.i. dans $U$.

(ii) Il existe dans $U$ une base de la topologie fine composée des ouverts fins $\left(V_{\alpha}\right)_{\alpha \in I}$ de fermeture fine $\tilde{V}_{\alpha} \subset U(\alpha \in I)$, telle que:

$$
f(x) \geqslant E_{x}\left(f\left(X_{\tau_{V \alpha}}\right) ; \tau_{V \alpha}<\zeta\right) \quad \text { pour tout } x \in V_{\alpha}(\alpha \in I) .
$$

LEMME 5. Soit $g: E \rightarrow[0,+\infty]$ une fonction semi-continue supérieurement (s.c.s.) pour la topologie cofine de $E$ et $\grave{a}$ valeurs finies sur l'ensemble $e \subset E$. Alors:

$$
R_{g}^{e}(x)=\inf \left\{R_{g}^{W}(x): W \supset e, \text { cofinement ouvert }\right\}
$$

où $R_{g}^{A}$ dénote la réduite de $g$ sur le sous-ensemble $A$ de $E$.

Démonstration. Soit $s$ une fonction surharmonique de $(\mathscr{H}, E)$, à valeurs finies et $>0$ dans $E$. Soit $v$ une fonction hyperharmonique, non-négative telle que $v \geqslant \mathrm{~g}$ dans $e$. Pour tout réel $\varepsilon>0$, l'ensemble $G_{\varepsilon}:\{x \mid v(x)+\varepsilon s(x)>g(x)\}$ est un voisinage ouvert cofin de $e$ puisque les fonctions $v$ et $s$ sont s.c.i. pour la topologie initiale de $E$. Ainsi:

$$
\inf \cdot R_{g}^{G_{\varepsilon}}(x) \leqslant \inf (v+\varepsilon s)(x)=v(x) .
$$

THÉORÈme 5 (principe du minimum fin). Soit $f$ une fonction finement hyperharmonique dans un ensemble finement ouvert $U$ de $E$. Supposons que:

1) $\underset{x \in U, x \rightarrow y}{\liminf \text {-fine }} f(x) \geqslant 0$ pour q.p. y sur $\partial_{\text {cof }} U \cap(\complement U)^{\text {cor }}$

2) liminf-cofine $f(x)>-\infty$ pour p.s. $r(d y)$ sur

$$
\left\{\partial_{\text {cof }} U \backslash(\complement U)^{\text {cor }}\right\} \cup\{(p=+\infty) \cap U\}
$$

3) $f \geqslant-p$ dans $U$ où $p(x)=\int u(x, y) r(d y)$ est un potentiel dans $E$. Alors $f$ est non-négative dans $U$. 
Démonstration. Soit $U_{0}$ l'intérieur cofine de $U$, alors d'une part $U \backslash U_{0}$ est un ensemble polaire $e_{0}([1], 4.1$.) et d'autre part tout point de $e_{0}$ appartient à $(\llbracket U)^{\mathrm{cor}}=\left(\left(U^{\prime}\right)^{\mathrm{cor}}\right.$, nous pouvons donc supposer dans le théorème que $U$ est à la fois finement et cofinement ouvert. Notons par $e$ l'ensemble polaire:

$$
\left\{y \mid y \in\left\{\partial_{\text {cof }} U \backslash(\complement U)^{\mathrm{cor}}\right\} \cup\{(p=+\infty) \cap U\} \text { et liminf-cofine } f(x)>-\infty\right\} .
$$

Définissons ensuite la fonction $\bar{f}: E \rightarrow[-\infty,+\infty]$ comme suit:

$$
\bar{f}:= \begin{cases}f \text { dans } U \backslash e & \\ \operatorname{liminf-cofine}_{x \in U, x \rightarrow y} f(x) & \text { pour tout } y \in e, \\ 0 & \text { partout ailleurs. }\end{cases}
$$

Alors $\bar{f}$ est q.p. finement s.c.i. dans $E$ et $>-\infty$ dans $U \backslash e$. D'autre part la fonction $g:=\bar{f}^{-}=\inf (-f, 0)$ est cofinement s.c.s. et à valeurs finies sur $e$, on a d'après le lemme 5 :

(14) $\inf \left\{R_{g}^{W}(x): W \supset e, W\right.$ cofinement ouvert $\}=0 \quad$ pour $x \in U \backslash e$.

Prenons un tel ensemble $W$ et posons $U^{\prime}=U \backslash \tilde{W}^{f}$ alors la restriction de $\bar{f}$ à $U^{\prime}$ vérifie les hypothèses du théorèmes 4 . Les conditions 1)-4) sont faciles à vérifier, il nous reste à vérifier 5). $\partial_{\text {cof }} U^{\prime}=\left(\partial_{\text {cof }} U^{\prime} \cap \partial_{\text {cof }} U\right) \cup$ $\left(\partial_{\text {cof }} U \backslash \partial_{\text {cof }} U^{\prime}\right)$, d'une part si $y$ est un point de $\partial_{\text {cof }} U \backslash\left(\complement U^{\prime}\right)^{\text {cor }}$ alors $y$ appartient aussi à $\partial_{\text {cof }} U \backslash\left(\complement U^{\prime}\right)^{\text {cor }}$ donc d'après 2) ces points $y$ appartiennent p.s. $r(d y)$ à $e$, ce qui entraine que $\left(\partial_{\text {cof }} U^{\prime} \cap \partial_{\text {cof }} U\right) \backslash\left(\mathcal{C} U^{\prime}\right)^{\text {cor }}$ est de mesure $r(d y)$ nulle. D'autre part l'ensemble $\left(\partial_{\text {cof }} U^{\prime} \backslash \partial_{\text {cof }} U\right)$ est contenue p.s. $r(d y)$ dans $U \backslash e$, donc $r(d y)$ ne charge pas les sous-ensembles polaires de $\partial_{\text {cof }} U^{\prime} \backslash \partial_{\text {cof }} U$ puisque $p(x)$ est fini dans $U \backslash e([3 \mathrm{~b}])$. Le théorème 4 donne donc:

$$
\bar{f}(x) \geqslant E_{x}\left(\bar{f}\left(X_{\tau_{U^{\prime}}}\right) ; \tau_{U}<\zeta\right) \quad \text { q.p. } x \text { dans } U^{\prime} .
$$

La fonction $f$ est finement hyperharmonique dans $U$ donc $y$ est finement continue ([3a], remarque du théorème 7), d'autre part le second membre de (15) est une fonction finement s.c.s. dans $U^{\prime}$ ([3c], proposition 5), on en déduit que (15) est vraie pour tout $x$ dans $U^{\prime}$. Mais on a aussi:

$$
\begin{aligned}
E_{x}\left(\bar{f}\left(X_{\tau_{U^{\prime}}}\right) ; \tau_{U^{\prime}}<\zeta\right) & =E_{x}\left(\vec{f}\left(X_{\tau_{U}}\right) ; \tau_{U}<T_{W}\right)+E_{x}\left(\bar{f}\left(X_{T_{W}}\right) ; \tau_{U}=T_{W}<\zeta\right) \\
& \geqslant E_{x}\left(\bar{f}\left(X_{\tau_{U}}\right) ; \tau_{U}<T_{W}\right)-R_{g}^{W}(x) .
\end{aligned}
$$

En faisant $W$ variant suivant le filtre des voisinages cofinement ouverts de $e$, on obtient enfin: 


$$
\begin{aligned}
E_{x}\left(\bar{f}\left(X_{\tau_{U^{\prime}}}\right) ; \tau_{U^{\prime}}<\zeta\right) & \geqslant \limsup _{W}\left(E_{x}\left(\bar{f}\left(X_{\tau_{U}}\right) ; \tau_{U}<T_{W}\right)-R_{g}^{W}(x)\right) \\
& \geqslant \liminf _{W}\left(E_{x}\left(\bar{f}\left(X_{\tau_{U}}\right) ; \tau_{U}<T_{W}\right)-\liminf _{W} R_{g}^{W}(x) .\right.
\end{aligned}
$$

On a donc d'après (14) et (15) :

$$
f(x) \geqslant E_{x}\left(f\left(X_{\tau_{U}}\right) ; \tau_{U}<\zeta\right) \quad \text { pour tout } x \text { de } U^{\prime} .
$$

Puisque $W$ a été choisi abitrairement et puisque $\bar{f}\left(X_{\tau_{U}}\right)=0$ p.s. $P^{x}(x \in U)$, on a ainsi:

$$
f(x) \geqslant 0 \quad \text { pour tout } x \text { de } U \backslash e .
$$

D'autre part dûe à l'inégalité (13) $f$ est aussi non-négative dans $e$.

Remarques. 1) Si les topologies fine et cofine de $E$ se coïncident, puisque $f$ est supposée $>-\infty$ dans $U$ l'assumption 2) du théorème 5 se réduit à:

$$
\text { liminf-fine } f(x)>-\infty \quad \text { p.s. } r(d y) \text { sur } \partial_{f} U \backslash(\complement U)^{\mathrm{r}}
$$

En particulier le théorème 5 se réduit pour le cas $\left(A_{2}\right)$ de $M^{\text {me }}$ Hervé avec l'axiome $(D)$ ou pour le cas classique d'un espace de Green à un résultat anoncé par B. Fuglede (communication privée)

2) Il est intéressant de se demander que si la condition:

$$
\underset{x \in U, x \rightarrow y}{\liminf \text {-cofine }} f(x)>-\infty \quad \text { pour tout } y \text { de }\{p=+\infty\} \cap U
$$

est superflue. Dans le cas du mouvement uniforme sur la droite réelle le résultat expecté est faux, ce qui nous amène à poser la question suivante: Supposons de plus que l'axiome $(D)$ est vrai pour l'espace harmonique donné. Soit $g: E \rightarrow[-\infty,+\infty]$ une fonction finement s.c.i. et $>-\infty$ en un point polaire $x_{0}$, peut-on montrer que

$$
\underset{x \in U, x \rightarrow x_{0}}{\liminf } \underset{f(x)>-\infty}{ }>-\infty
$$

\section{RÉFÉRENCES}

[1] R. M. Blumenthal and R. K. Getoor: Dual processes and potential theory, Proc. Twelfth Bienmial Sem. Canad. Math. Congr. (ed by R. Pyke), 1970, 37-56.

[2] B. Fuglede: Finely harmonic functions, Lecture Notes in Math. Bd. 289, 1972.

[3a] Nguyen-Xuan-Loc: Characterization of excessive functions on finely open, nearly Borel sets, Math. Ann., 196 (1972), 250-268.

[3b] - : Sur les potentiels semi-bornés, C.R. Acad. Sci. Paris Sér A-B, 27 (1972), $767-770$. 
[3c] — : Fine boundary minimum principle and dual processes, Z. Wahrscheinlichkeitstheorie und Verw. Gebiete, 27, 1973, 233-256.

[4a] P. A. Meyer: Probabilités et potentiel, Hermann, Paris 1966.

[4b] _ _ : Processus de Markov: la frontière de Martin, Lecture Notes in Math. Bd. 77, 1968.

Mathematisches Institut

der Universität Erlangen-Nürnberg 